ball pass before and after a high-speed $180^{\circ}$ pivot turn on the force plates.

Following variables were analysed

Peak vertical ground reaction force $(\mathrm{N} / \mathrm{kg})$, peak trunk lateral flexion angle (degree), peak knee flexion angle (degree), peak knee valgus angle (degree), peak knee flexion moment $(\mathrm{Nm} / \mathrm{kg})$, peak knee abduction moment $(\mathrm{Nm} / \mathrm{kg})$, and peak knee internal and external rotation moments $(\mathrm{Nm} / \mathrm{kg})$. Mean of three trials was used. Injuries and exposure were registered for 12 months.

Main Outcome Measurements New acute non-contact knee injury.

Results The complete data was obtained from 109 female and 149 male basketball $(n=130)$ and floorball $(n=128)$ players. A total of 18 new non-contact knee injuries were registered $(0.3$ injuries/1000 h of exposure). Female players sustained 14 knee injuries and male players 4 . A higher rate of knee injuries was observed in females compared with males (incidence rate ratio 6.2, 95\% CI 2.1-21.7). Eight ACL injuries were registered (all in female players). Females displayed significantly larger peak knee valgus angles compared with male (mean for female and male, respectively: 13.9 and 2.0 degree). No significant associations between biomechanical variables and knee injury risk were found.

Conclusions Female players were at increased risk of knee and ACL injury compared with males. Female players performed the 180-degree pivot turn with significantly larger knee valgus compared with male. However, none of the investigated variables were associated with knee injury risk in youth basketball and floorball players.

\section{INJURY BURDEN DIFFERS CONSIDERABLY BETWEEN SINGLE TEAMS FROM GERMAN PROFESSIONAL MALE HANDBALL: SURVEILLANCE OF THREE CONSECUTIVE SEASONS}

${ }^{1}$ Hendrik Bloch, ${ }^{1}$ Christian Klein, ${ }^{2}$ Patrick Luig. ${ }^{1}$ VBG (German Statutory Accident Insurance for the Administrative Sector, Department of Sports Injury Prevention), Hamburg, Germany; ${ }^{2}$ DHB (German Handball Federation), Dortmund, Germany

\subsection{6/bjsports-2021-IOC.41}

Background Although high injury prevalences and incidences for professional handball were reported, longitudinal analysis of injury burden remain rare.

Objective Analysis of the injury data of the national statutory accident insurance (VBG) for the two highest divisions in German professional male handball over three consecutive seasons regarding interseason, inter-division and inter-team differences of injury burden.

Design Prospective observational open cohort study over the seasons 2014/2015, 2015/2016 and 2016/2017.

Setting German professional male handball (Handball Bundesli$\mathrm{ga}=\mathrm{HB}, 2$. Handball Bundesliga $=\mathrm{HB} 2$ ).

Patients (or Participants) All first and second division handball players who played at least one competitive club match within the seasons $2014 / 15 \quad(n=808), \quad 15 / 16 \quad(n=757)$ and $16 / 17$ $(n=758)$ were included.

Interventions (or Assessment of Risk Factors) Analysis of all injuries that were registered by clubs or physicians with the German statutory accident insurance for professional athletes (VBG) as part of occupational accident reporting and that led to time loss and/or to medical attention.
Main Outcome Measurements Inter-team comparisons were calculated using the following formula: 'Cumulative injury burden resulting from mandatory reportable injuries $(\geq 4$ days) per team-season/number of competitive matches per teamseason'.

Results Every German professional handball player missed on average (cumulative) 28.7 days per season due to injuries. The total burden equalled 66,597 days of absence (HB: 31,247, HB2: 35,350). The mean injury burden per competitive match over the three observed seasons for all $\mathrm{HB}$ teams ranged from 12.5 to 14.2 days of absence with individual rates ranging from 4.3 to $28.8(2014 / 2015), 2.1$ to 27.5 $(2015 / 2016)$ and 2.3 to 33.6 (2016/2017). For HB2 teams the mean burden ranged from 13.3 to 14.6 days of absence per competitive match, with individual rates ranging from 3.0 to $25.1(2014 / 2015), 2.5$ to $30.0(2015 / 2016)$ and 0.9 to $35.7(2016 / 17)$.

Conclusions These wide ranges indicate that a reduction in the injury burden seems generally possible and that some teams and coaching staffs are more successful concerning their preventive (coach, medical and therapeutic specific) player support.

\section{EVALUATION OF BODY CHECKING POLICY FOR INJURY PREVENTION IN NON-ELITE ADOLESCENT ICE HOCKEY PLAYERS}

1,2,3,4,5,6 Carolyn Emery, 1,2,3Paul H Eliason, 'Warriyar KV Vineetha, 1,4Luz PalaciosDerflingher, ${ }^{1,2,3}$ Amanda M Black, ${ }^{1}$ Maciej Krolikowski, ${ }^{1}$ Nicole Spencer, 1,2,3,7,8 Kathryn Schneider, ${ }^{9}$ Shelina Babul, ${ }^{10}$ Martin Mrazik, ${ }^{11,12}$ Constance Lebrun, ${ }^{13}$ Claude Goulet, ${ }^{14}$ Alison K MacPherson, $1,3,4,5,6$ Brent E Hagel. ${ }^{1}$ Sport Injury Prevention Research Centre, Faculty of Kinesiology, University of Calgary, Calgary, Canada; ${ }^{2}$ Hotchkiss Brain Institute, Cumming School of Medicine, University of Calgary, Calgary, Canada: ${ }^{3}$ Alberta Children's Hospital Research Institute, Cumming School of Medicine, University of Calgary, Calgary, Canada; ${ }^{4}$ Community Health Sciences, Cumming School of Medicine, University of Calgary, Calgary, Canada; ${ }^{5}$ Department of Pediatrics, Cumming School of Medicine, University of Calgary, Calgary, Canada; ${ }^{6} \mathrm{O}^{\prime}$ Brien Institute for Public Health, Cumming School of Medicine, University of Calgary, Calgary, Canada; 'Sport Medicine Centre Acute Sport Concussion Clinic, University of Calgary, Calgary, Canada; ${ }^{8}$ Evidence Sport and Spinal Therapy, Calgary, Canada, ${ }^{9}$ British Columbia Injury Research and Prevention Unit, Department of Pediatrics, University of British Columbia, Vancouver, Canada; ${ }^{10}$ Faculty of Education, University of Alberta, Edmonton, Canada; ${ }^{11}$ Department of Family Medicine, Faculty of Medicine and Dentistry, University of Alberta, Edmonton, Canada; ${ }^{12}$ Glen Sather Sports Medicine Clinic, University of Alberta, Edmonton, Canada; ${ }^{13}$ Department of Physical Education, Faculty of Education, Université Laval, Quebec City, Canada; ${ }^{14}$ Faculty of Health, York University, Toront, Canada

\section{$10.1136 /$ bjsports-2021-IOC.42}

Background Body checking is associated with an increased risk of injury and concussion in Pee Wee (ages 11-12) and nonelite (lower $70 \%$ by division of play) Bantam (ages 13-14) ice hockey players. This research informed local and provincial policy changes disallowing body checking in non-elite Midget (ages 15-17).

Objective To evaluate the rate ratios of game-related injury and concussion in non-elite Midget players following policy change disallowing body checking in games compared with similar leagues still allowing body checking.

Design Prospective cohort.

Setting Community ice hockey rinks.

Participants Non-elite Midget ice hockey players from 44 teams (453 player-records) where policy disallowed body checking in the lower $70 \%$ of divisions of play, and 52 teams (674 player-records) from similar divisions where policy 